\title{
Diagnosing and classifying malignant lymphomas is improved by referring cases to a panel of expert pathologists
}

\author{
S. A. M. van de Schans • L. Strobbe • I. M. van der Holst • \\ J. Meijer • V. Mattijssen • I. M. de Kievit • C. Mandigers • \\ J. Raemaekers • K. K. H. Aben • J. H. van Krieken
}

Received: 3 June 2013 / Accepted: 3 October 2013 / Published online: 24 October 2013

(C) Springer-Verlag Berlin Heidelberg 2013

\begin{abstract}
Pathological classification of lymphomas can be difficult. The aim of the present study was to evaluate the value of referring cases to a pathology panel. All patients diagnosed with a primary lymphoma in 2000 or 2001 were selected from the regional population-based cancer registry. A panel of at least three expert pathologists reviewed all pathological diagnoses in joint sessions using a multiheaded microscope. The discordance between the initial diagnosis and the
\end{abstract}

S. A. M. van de Schans $(\varangle) \cdot$ I. M. van der Holst $\cdot$ K. K. H. Aben Department of Registry and Research, Comprehensive Cancer Centre The Netherlands, Utrecht, PO Box 1281, 6501 BG Nijmegen, The Netherlands

e-mail: sam_vd_schans@hotmail.com

L. Strobbe $\cdot$ J. Raemaekers

Department of Haematology, Radboud University Medical Centre, Nijmegen, The Netherlands

J. Meijer

Department of Pathology, Rijnstate Hospital, Arnhem,

The Netherlands

V. Mattijssen · J. Raemaekers

Department of Haematology, Rijnstate Hospital, Arnhem,

The Netherlands

I. M. de Kievit

Department of Pathology, Canisius-Wilhelmina Hospital, Nijmegen, The Netherlands

C. Mandigers

Department of Haematology, Canisius-Wilhelmina Hospital, Nijmegen, The Netherlands

K. K. H. Aben

Department of Epidemiology, Biostatistics and HTA, Radboud University Medical Centre, Nijmegen, The Netherlands

J. H. van Krieken

Department of Pathology, Radboud University Medical Centre,

Nijmegen, The Netherlands expert panel was determined. In addition, survival analyses were done. The discordance rate between the initial and panel diagnosis was $14 \%$. For cases actively referred to the panel $(N=161)$, the discordance rate was 11 versus $16 \%$ for cases not actively referred to the panel $(N=183)$; however, this difference is not statistically significant. There was no statistically significant difference in survival between patients with a concordant diagnosis compared to patients with a discordant diagnosis, but there are differences in the types of lymphomas between these. This population-based study showed that the expert panel changed the diagnosis in almost one of seven lymphoma diagnoses. The diagnoses of cases not actively referred were not significantly less often changed compared to those from cases actively referred. This indicates that the percentage of discordant cases was not increased by referral bias. We conclude that referral of lymphoma cases to a diagnostic panel results in improved diagnosis.

Keywords Diagnosis - Classification · Pathology · Lymphoma $\cdot$ Panel $\cdot$ Evaluation

\section{Introduction}

Cancers arising from lymphocytes are a heterogeneous group of malignancies with various clinical and biological features. According to the World Health Organization (WHO) classification of hematopoietic and lymphoid tissue fourth edition [1], the lymphoid cancers are divided into precursor, mature B cell (Hodgkin and non-Hodgkin lymphoma) and mature $\mathrm{T}$ and NK cell neoplasms. Based on clinical features, histopathology, immunophenotype and genetic criteria, these main diagnostic groups are further subdivided into a large variety of separate entities. This classification was introduced in 2001 (and revised in 2008) after a decade with numerous clinical and 
pathologic schemes used to classify lymphomas and lymphoid leukemias $[2,3]$.

For optimal patient management, it is important to classify lymphomas correctly and according to the latest classification, but this is a complex process $[1,4,5]$. Histologic sections and immunostains must be of high quality, and considerable knowledge on and experience with the pathology of lymphoproliferations is required. In addition, molecular and cytogenetic tests have to be available to identify several subtypes of lymphoma [1,6]. Earlier studies have shown that interobserver disagreement in classifying lymphoma exists (6-32\%) even between expert hemato-pathologists [4-9]. Revision of diagnoses by a panel of pathologists performed in various countries showed that the lowest rate of discordance was found for diffuse large B cell lymphoma and nodular sclerosis classical Hodgkin lymphoma whereas the highest discordance was reported for follicular lymphoma grade III because of the difficulties with grading of follicular lymphomas $[4,7-9]$.

In 2000, in the Eastern part of the Netherlands, a lymphoma panel of expert pathologists was initiated to review the pathology from patients with a primary lymphoma diagnosis. The panel was installed to standardize and improve the pathological classification of lymphomas in this part of the country. The aim of the present study was to evaluate the value of this pathology panel. Discordance rates between the diagnoses of the initial pathologists and the expert panel were determined, and the effect of discordant diagnoses on survival was evaluated.

\section{Material and methods}

\section{Study population and data collection}

Patients with malignant lymphoma were identified through the regional population-based cancer registry in the eastern part of the Netherlands. All patients diagnosed with a primary lymphoma diagnosed between January 1, 2000 and December 31,2001 , in one of seven hospitals within the catchment area was selected. Date of initial diagnosis, date of birth, gender, pathological diagnosis and vital status were retrieved from the cancer registry. Follow-up concerning vital status was completed by annual linkage to the nationwide municipality network (GBA), which stores information on all Dutch inhabitants including vital status.

Three pathology laboratories, including one academic, performed the original diagnoses of all patients from the abovementioned seven hospitals. The pathology panel consists of at least three pathologists with special training in haematopathology, one pathologist per pathology laboratory. Thus, sometimes, the pathologist who made the initial diagnosis reviewed his or her own case, but then the two other experts of the panel confirmed or rejected the initial diagnosis. At a multiheaded microscope, all original slides stained with haematoxylin and eosin and the immunohistochemical stained sections were reviewed once every other week. Clinical information on age, location of biopsy and clinical and pathological history of the patient was available for the panel from the hospital of first diagnosis. After simultaneous review by all expert pathologists, a consensus diagnosis was formulated; occasionally, further tests were ordered after which the case was reviewed again. The final classification was not based on voting, but agreed by reconciliation. Feedback concerning the discordant cases was given to the initial pathologist. Cases referred to one of the two haematopathologists in the academic centre for consultation before review in the expert panel were excluded from this study.

More than 30 different lymphoma entities following the WHO classification were included in this study (Table 1). We categorized these different entities into 17 lymphoma groups. The groups were defined as B lymphoblastic lymphoma (B LBL), chronic lymphocytic leukaemia/small lymphocytic lymphoma (CLL), lymphoplasmacytic lymphoma (LPL), plasmacytoma (PC), marginal zone lymphoma (MZL), follicular lymphoma grades I and II (FL 1-2), follicular lymphoma grade III (FL 3), mantle cell lymphoma (MCL), diffuse large B cell lymphoma (DLBCL), Burkitt lymphoma (BL), T cell and NK cell lymphomas (TCL), nodular lymphocyte predominant Hodgkin lymphoma (NLPHL), classical Hodgkin lymphoma (CHL), lymphoproliferative disease associated with immunodeficiency (LPD IDA), lymphoma with transformation (lymphoma with a low-grade and high-grade component) (LT), low-grade lymphoma not otherwise specified (LL NOS) and lymphomas not otherwise specified (L NOS). When the panel diagnosis resulted in a different group compared to the initial diagnosis, we considered this as a relevant change and as a discordant diagnosis. The definition of these groups (Table 1) was discussed within the panel and evaluated by haematologists. They concluded that relevant changes (i.e. discordant diagnoses/changes between groups) were defined as changes with consequences for additional diagnostic procedures, therapy or prognosis. Discordant diagnoses included alterations in type of lymphoma, for example, Hodgkin lymphoma to non-Hodgkin lymphoma or in histological subtypes, e.g. chronic lymphocytic leukaemia to follicular lymphoma but also changes in the grade of follicular lymphoma or when one of the two diagnoses was not specific (see Table 3 for other changes between groups). A change in subtype within the group of diffuse large B cell lymphoma or the group of classical Hodgkin lymphoma was not considered a relevant change because this would not result in alterations in additional diagnostics, treatment and prognosis.

The pathology reviews were performed at different moments in time. A part of the cases was actively referred by the initial pathologist to the pathology panel. Another part of the 
Table 1 Grouping of WHO lymphoma types included in pathology revision by the panel of expert pathologists

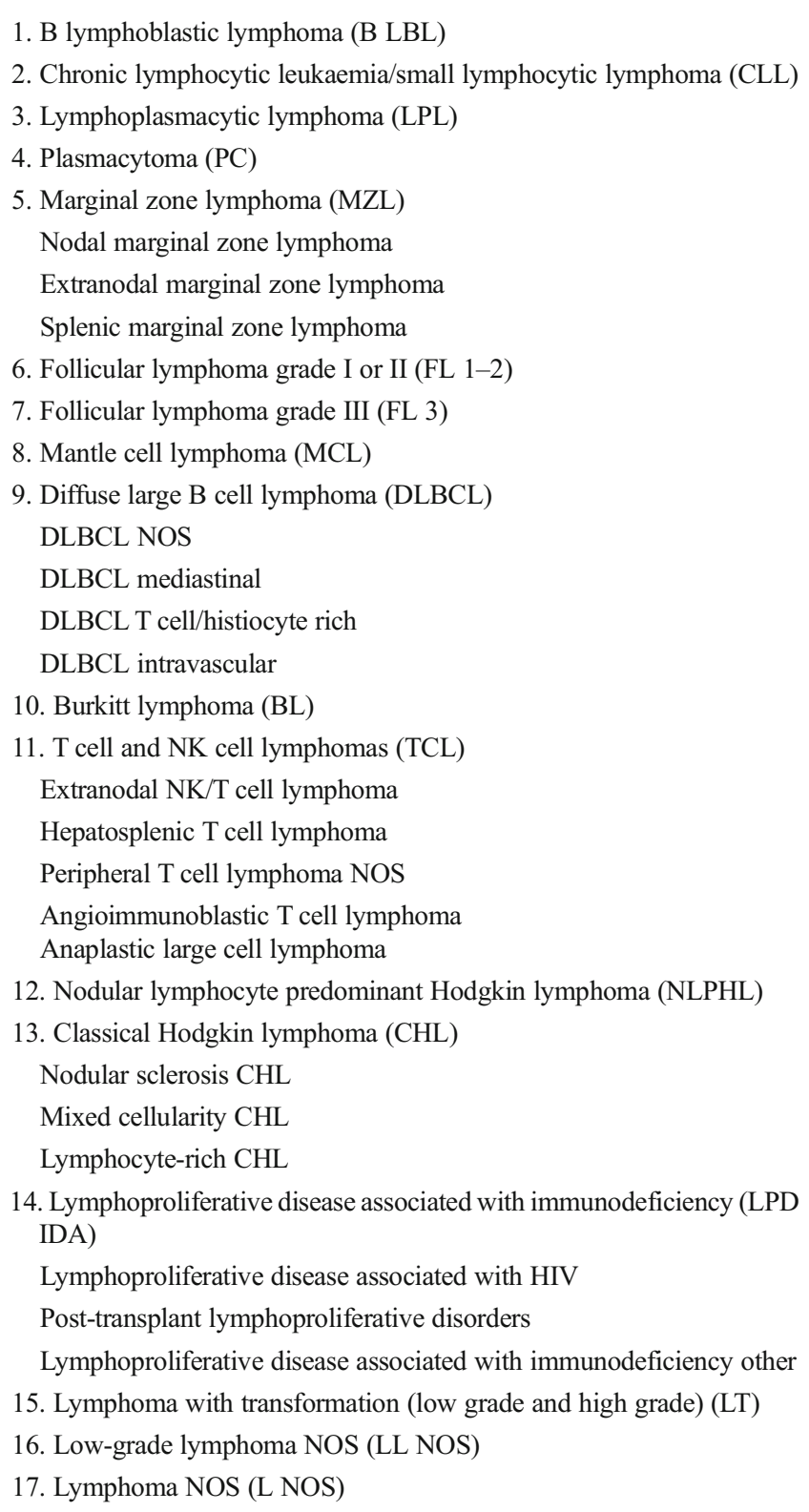

NOS not otherwise specified

cases was initially not referred to the panel, and the biopsies of these patients were revised by the panel members for the present study. Although this review was almost 10 years after the initial diagnosis, no markers or criteria were used that were introduced in the mean time. The selection of cases that were actively referred to the panel was up to the referring pathologists and differs between the three pathology laboratories. Reasons for referring a case could be a difficult diagnosis or a special interest in haematology, and therefore, more awareness of the panel or interest in the opinion of the expert panel or policy of the pathology laboratory to send in all cases.
Patients with a cutaneous lymphoma were excluded because in the Netherlands, these patients are being reviewed in a national panel. Patients diagnosed at autopsy, patients of whom only a fine needle aspiration was available and patients of whom no pathology tissue could be retrieved were also excluded from this study.

Statistical analyses

Descriptive analyses were performed to characterize the study population. The discordance rate between initial and panel diagnosis was calculated. The $\mathrm{k}$ statistic, which represents the extent of agreement beyond that expected by chance, and the $95 \%$ confidence interval around this statistic was calculated [10]. The discordance rate was stratified to cases referred and cases not referred to the panel and histological subtype.

Follow-up time of all patients was calculated as time between date of initial diagnosis and date of death or date of end of study or data of end of follow-up. Overall survival was estimated with the Kaplan-Meier method, and survival curves were compared with the log-rank test. Overall survival was analyzed according to discordance/concordance and in the two subgroups (patients referred and patients not referred). All analyses were performed with SPSS 14.0 and SAS V8.2.

\section{Results}

In the period 2000 and 2001, 391 patients were diagnosed with a primary malignant lymphoma. Pathology tissue from 40 patients could not be retrieved; in four patients, the tumour appeared to be diagnosed at autopsy, and in three patients, only a fine needle aspiration was available; these patients were excluded. The date of panel review could not be retrieved for nine patients.

A description of the 344 patients and their tumour characteristics is presented in Table 2. Pathology laboratory B, serving three hospitals, was the largest laboratory and performed the diagnoses of $55 \%$ of all patients. Forty-seven percent of all cases were actively referred to the panel. The most common lymphoma type diagnosed by the panel was diffuse large B cell lymphoma (32\%). Lymphoma types were more or less similar in actively referred and not referred patients (Table 2).

In 47 patients (actively referred plus not referred), the initial diagnosis made by the referring pathologist was different from the panel diagnosis (discordance rate of $14 \%$; kappa $=0.84$; $95 \%$ CI, 0.78-0.89) (Table 3). The discordance rate was different for cases actively referred to the panel and cases not referred to the panel (11 vs. $16 \%$, respectively), although not statistically significant. The most frequent panel subtype diagnoses that were initially not correctly classified were FL 3 
Table 2 Patients' and lymphoma characteristics of cases referred to the panel not referred to the panel and the total population

\begin{tabular}{|c|c|c|c|}
\hline & Referred & Not referred & Total \\
\hline Number of patients & 161 & 183 & 344 \\
\hline \multicolumn{4}{|l|}{ Gender } \\
\hline Male & $99(61 \%)$ & $92(50 \%)$ & $191(56 \%)$ \\
\hline Female & $62(39 \%)$ & $91(50 \%)$ & $153(44 \%)$ \\
\hline \multicolumn{4}{|l|}{ Pathology laboratory } \\
\hline $\mathrm{A}$ & $39(24 \%)$ & $65(36 \%)$ & $104(30 \%)$ \\
\hline $\mathrm{B}$ & $72(45 \%)$ & $118(64 \%)$ & $190(55 \%)$ \\
\hline $\mathrm{C}$ & $50(31 \%)$ & - & $50(15 \%)$ \\
\hline \multicolumn{4}{|l|}{ Lymphoma subtypes $^{\mathrm{a}}$} \\
\hline B LBL & - & $1(0.5 \%)$ & $1(0.3 \%)$ \\
\hline CLL & $3(2 \%)$ & $9(5 \%)$ & $12(4 \%)$ \\
\hline LPL & - & $1(0.5 \%)$ & $1(0.3 \%)$ \\
\hline $\mathrm{PC}$ & $1(1 \%)$ & $1(0.5 \%)$ & $2(1 \%)$ \\
\hline MZL & $14(9 \%)$ & $11(6 \%)$ & $25(7 \%)$ \\
\hline FL 1-2 & $13(8 \%)$ & $25(14 \%)$ & $38(11 \%)$ \\
\hline FL 3 & $8(5 \%)$ & $7(4 \%)$ & $15(4 \%)$ \\
\hline$M C L^{b}$ & $11(7 \%)$ & $4(2 \%)$ & $15(4 \%)$ \\
\hline $\mathrm{DLBCL}^{\mathrm{c}}$ & $59(36 \%)$ & $52(28 \%)$ & $111(32 \%)$ \\
\hline $\mathrm{BL}$ & $3(2 \%)$ & $5(3 \%)$ & $8(2 \%)$ \\
\hline TCL & $8(5 \%)$ & $12(7 \%)$ & $20(6 \%)$ \\
\hline NLPHL & $4(2 \%)$ & $2(1 \%)$ & $6(2 \%)$ \\
\hline CHL & $17(11 \%)$ & $21(12 \%)$ & $38(11 \%)$ \\
\hline LPD IDA & $5(3 \%)$ & $10(6 \%)$ & $15(4 \%)$ \\
\hline LT & $7(4 \%)$ & $3(2 \%)$ & $10(3 \%)$ \\
\hline LL NOS & $2(1 \%)$ & $7(4 \%)$ & $9(3 \%)$ \\
\hline L NOS & $6(4 \%)$ & $12(7 \%)$ & $18(5 \%)$ \\
\hline
\end{tabular}

$B L B L$ B lymphoblastic lymphoma, $C L L$ chronic lymphocytic leukae$\mathrm{mia} / \mathrm{small}$ lymphocytic lymphoma, $L P L$ lymphoplasmacytic lymphoma, $P C$ plasmacytoma, $M Z L$ marginal zone lymphoma, $F L$ 1-2 follicular lymphoma grades I and II, $F L 3$ follicular lymphoma grade III, $M C L$ mantle cell lymphoma, $D L B C L$ diffuse large B cell lymphoma, $B L$ Burkitt lymphoma, TCL T cell and NK cell lymphomas, NLPHL nodular lymphocyte predominant Hodgkin lymphoma, $C H L$ classical Hodgkin lymphoma, $L P D I D A$ lymphoproliferative disease associated with immunodeficiency, $L T$ lymphoma with transformation (lymphoma with a lowgrade and high-grade component), LL NOS low-grade lymphoma not otherwise specified, L NOS lymphomas not otherwise specified

${ }^{a}$ Lymphoma subtypes: subtypes are shown by the diagnosis of the panel of experts and not the initial diagnose because the panel diagnose was used as gold standard

${ }^{\mathrm{b}}$ One patient in this group was diagnosed with two tumours, as well as the MZL a CLL was diagnosed

${ }^{\mathrm{c}}$ One patient in this group was diagnosed with two tumours, as well as the DLBCL a T cell lymphoma was diagnosed. One patient in this group was diagnosed with two tumours, as well as the DLBCL a classic Hodgkin lymphoma was diagnosed

(33 \%), LT (90 \%), LL NOS (44 \%) and L NOS (61\%). The discordance of the initial pathologist and the expert panel diagnosis was lowest for B LBL, LPL, PC, MCL, DLBCL,
Table 3 Discordance rate of cases referred to the panel, cases not referred to the panel and the total population

\begin{tabular}{llll}
\hline & Referred $^{\mathrm{a}}$ & Not referred $^{\mathrm{a}}$ & Total \\
\hline Number of patients & 161 & 183 & 344 \\
Discordance (N) & 18 & 29 & 47 \\
Discordance rate & $11 \%$ & $16 \%$ & $14 \%$ \\
\hline
\end{tabular}

${ }^{a}$ Difference between actively referred and not referred patients was not statistically significant $(p=0.2)$

TCL and HL (Table 4). Discordance rate varied between 11 and $23 \%$ between laboratories.

The characteristics of patients with a discordant diagnosis were different from patients with a concordant diagnosis. Patients with a discordant diagnosis were older (median age of 68 vs. 63), and the distribution of NHL subtypes was different: less DLBCL (9 vs. $36 \%$ ), more LL NOS (9 vs. $2 \%$ ), more FL 3 (11 vs. $3 \%$ ), less TCL ( 0 vs. $7 \%$ ), less HL (4 vs. $12 \%$ ) and more L NOS (23 vs. $2 \%$ ).

In Fig. 1, the overall survival of patients with a concordant versus discordant diagnosis is presented. There was no statistically significant difference in survival between patients with a concordant diagnosis compared to patients with a discordant diagnosis [5-year survival $48 \%$ (95\% CI $42-53 \%)$ vs. $53 \%$ (95\% CI 39-67\%)]. Five-year survival of $47 \%$ for patients actively referred and $50 \%$ for patients not actively referred to the panel. Referred patients with a discordant diagnosis seem to have a lower but not significant survival than not referred patients (Fig. 2).

\section{Discussion}

Our population-based study showed that in $14 \%$ of all lymphoma cases, the original diagnosis of the referring pathologist differed from the diagnosis made by the panel of expert pathologists. For cases actively referred to the panel (47\%), the discordance rate was 11 versus $16 \%$ for cases not actively referred to the panel; this was however not statistically significant. The majority of lymphomas not correctly classified by the referring pathologist included follicular lymphomas grade III, lymphomas with both low- and high-grade component, low-grade lymphoma not otherwise specified and lymphomas not otherwise specified. There was no statistically significant difference in survival between patients with a concordant diagnosis compared to patients with a discordant diagnosis.

The discordance rate reported in our study was similar to rates observed in other studies. For example, the International Lymphoma Study Group (ILSG) started in 1997 the nonHodgkin's Lymphoma Classification Project to determine the intraobserver reproducibility of the new classification of 
Table 4 Overview of discordance between initial and panel diagnosis

\begin{tabular}{|c|c|c|c|c|c|c|c|c|c|c|c|c|c|c|c|c|c|c|}
\hline \multirow[t]{2}{*}{ Initial diagnosis } & \multicolumn{18}{|c|}{ Panel diagnosis } \\
\hline & 1 & 2 & 3 & 4 & 5 & 6 & 7 & 8 & 9 & 10 & 11 & 12 & 13 & 14 & 15 & 16 & 17 & Total \\
\hline 1. B LBL & 1 & 0 & 0 & 0 & 0 & 0 & 0 & 0 & 0 & 0 & 0 & 0 & 0 & 0 & 0 & 0 & 0 & 1 \\
\hline 2. CLL & 0 & 10 & 0 & 0 & 1 & 0 & 0 & 0 & 0 & 0 & 0 & 0 & 0 & 0 & 1 & 0 & 1 & 13 \\
\hline 3. LPL & 0 & 0 & 1 & 0 & 1 & 0 & 0 & 0 & 0 & 0 & 0 & 0 & 0 & 0 & 0 & 0 & 0 & 2 \\
\hline 4. PC & 0 & 0 & 0 & 2 & 0 & 0 & 0 & 0 & 0 & 0 & 0 & 0 & 0 & 0 & 0 & 0 & 0 & 2 \\
\hline 5. MZL & 0 & 0 & 0 & 0 & 21 & 2 & 0 & 0 & 2 & 0 & 0 & 0 & 0 & 0 & 0 & 0 & 1 & 26 \\
\hline 6. FL 1-2 & 0 & 0 & 0 & 0 & 0 & 35 & 5 & 0 & 0 & 0 & 0 & 0 & 1 & 0 & 1 & 2 & 1 & 45 \\
\hline 7. FL 3 & 0 & 1 & 0 & 0 & 1 & 1 & 10 & 0 & 0 & 0 & 0 & 0 & 0 & 0 & 0 & 1 & 0 & 14 \\
\hline 8. MCL & 0 & 0 & 0 & 0 & 0 & 0 & 0 & 15 & 1 & 0 & 0 & 0 & 0 & 0 & 0 & 0 & 0 & 16 \\
\hline 9. DLBCL & 0 & 0 & 0 & 0 & 0 & 0 & 0 & 0 & 107 & 1 & 0 & 0 & 1 & 1 & 7 & 0 & 4 & 121 \\
\hline 10. BL & 0 & 0 & 0 & 0 & 0 & 0 & 0 & 0 & 0 & 7 & 0 & 0 & 0 & 0 & 0 & 0 & 0 & 7 \\
\hline 11. TCL & 0 & 0 & 0 & 0 & 0 & 0 & 0 & 0 & 0 & 0 & 20 & 0 & 0 & 0 & 0 & 0 & 0 & 20 \\
\hline 12. NLPHL & 0 & 0 & 0 & 0 & 0 & 0 & 0 & 0 & 0 & 0 & 0 & 6 & 0 & 1 & 0 & 0 & 0 & 7 \\
\hline 13. CHL & 0 & 0 & 0 & 0 & 0 & 0 & 0 & 0 & 0 & 0 & 0 & 0 & 36 & 0 & 0 & 0 & 3 & 39 \\
\hline 14. LPD IDA & 0 & 0 & 0 & 0 & 0 & 0 & 0 & 0 & 0 & 0 & 0 & 0 & 0 & 13 & 0 & 0 & 1 & 14 \\
\hline 15. LT & 0 & 0 & 0 & 0 & 0 & 0 & 0 & 0 & 0 & 0 & 0 & 0 & 0 & 0 & 1 & 0 & 0 & 1 \\
\hline 16. LL NOS & 0 & 1 & 0 & 0 & 0 & 0 & 0 & 0 & 0 & 0 & 0 & 0 & 0 & 0 & 0 & 5 & 0 & 6 \\
\hline 17. L NOS & 0 & 0 & 0 & 0 & 1 & 0 & 0 & 0 & 1 & 0 & 0 & 0 & 0 & 0 & 0 & 1 & 7 & 10 \\
\hline Total & 1 & 12 & 1 & 2 & 25 & 38 & 15 & 15 & 111 & 8 & 20 & 6 & 38 & 15 & 10 & 9 & 18 & 344 \\
\hline$\%$ Discordance & 0 & 17 & 0 & 0 & 16 & 8 & 33 & 0 & 4 & 13 & 0 & 0 & 5 & 13 & 90 & 44 & 61 & 14 \\
\hline
\end{tabular}

Kappa is 0.84

$B L B L$ B lymphoblastic lymphoma, $C L L$ chronic lymphocytic leukaemia/small lymphocytic lymphoma, $L P L$ lymphoplasmacytic lymphoma, $P C$ plasmacytoma, $M Z L$ marginal zone lymphoma, $F L 1-2$ follicular lymphoma grades I and II, FL 3 follicular lymphoma grade III, $M C L$ mantle cell lymphoma, $D L B C L$ diffuse large B cell lymphoma, $B L$ Burkitt lymphoma, TCL T cell and NK cell lymphomas, NLPHL nodular lymphocyte predominant Hodgkin lymphoma, $C H L$ classical Hodgkin lymphoma, $L P D I D A$ lymphoproliferative disease associated with immunodeficiency, $L T$ lymphoma with transformation (lymphoma with a low-grade and high-grade component), LL NOS low-grade lymphoma not otherwise specified, $L$ NOS lymphomas not otherwise specified

non-Hodgkin's lymphoma (NHL) of patients' diagnoses in 1988-1990 in eight countries [4]. They concluded that when expert pathologists work from clear and agreed upon criteria, the diagnosis of NHL can be at least $85 \%$ accurate for most of

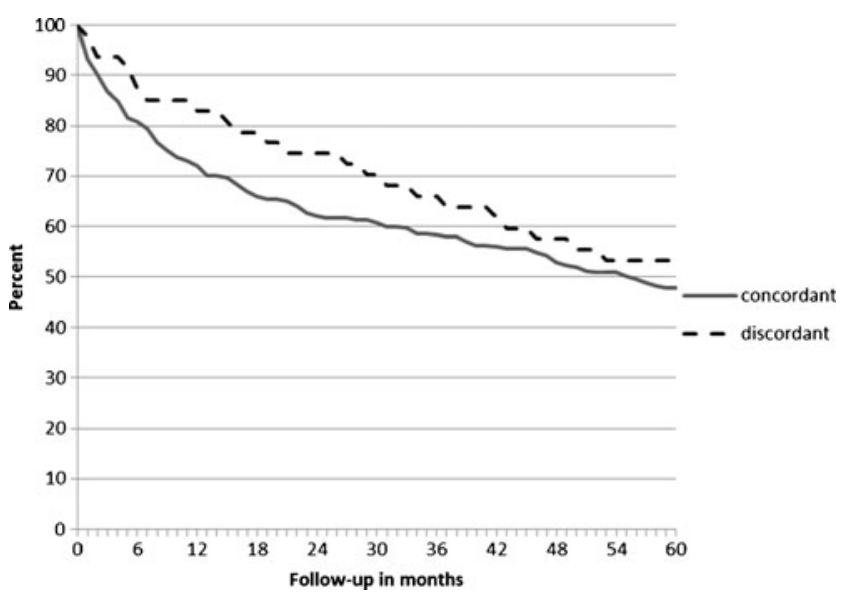

Fig. 1 Overall survival of lymphoma patients by concordant/discordant diagnoses the common types. Since then, the following studies reported on expert panels reviewing lymphoma diagnosis in their region. In Wales, a panel reviewing NHL patients diagnosed in 1998-2000 resulted in a discordance rate of $17 \%$ [5]. A panel reviewing NHL patients diagnosed in 2000 in India resulted in an $18 \%$ discordance rate [6]. In the USA, a study including only the five most common B cell NHL resulted in a lower discordance rate of $6 \%$, studying patients diagnosed in 2000 2004 [7]. Next to the fact that only five B cell NHL were included in the review process, different grading of follicular lymphoma was not considered discordant in this study, which probably accounts for the lower discordance rate in this study compared to our study. The North Central London Lymphoma network observed the discordance between referred diagnosis and expert diagnosis of NHL patient diagnosed between 2003 and 2008; the discordance improved significantly between 2003 and 2008 (32 to $13 \%$ ) [11]. This could indicate that feedback of the panel towards initial pathologists on discordant diagnosis improves the original diagnosis in the following years. This finding was also mentioned by the 


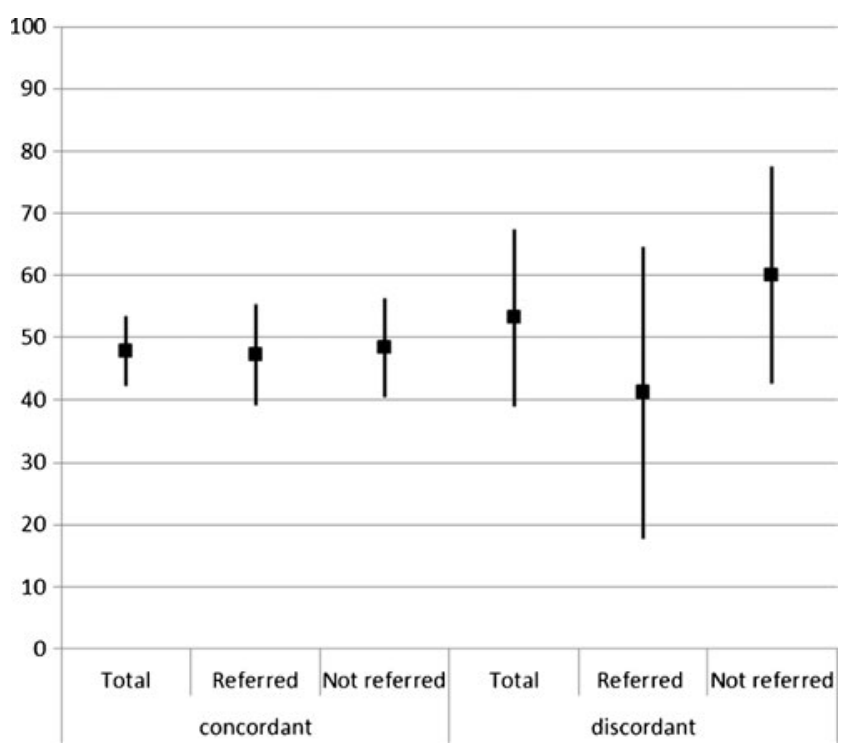

Fig. 2 5-year overall survival of lymphoma patients by concordant/ discordant diagnosis and referral (with $95 \% \mathrm{CI}$ )

participating pathologists in our region and will be investigated in further research.

The discordance rate in our study varied between NHL subtypes; follicular lymphomas grade III and lymphomas with both high- and low-grade aspects were more difficult to classify. Grading follicular lymphoma is problematical because it is difficult to estimate the number of centroblasts and centrocytes per high-power field. In our study, nine cases with a primary diagnosis of low-grade lymphoma or DLBCL were found in which the panel reported components of both low- and highgrade lymphoma. In these cases, the panel concluded transformed lymphoma. As patients with a transformed lymphoma have a worse prognosis compared to low-grade lymphoma or primary DLBCL, it is important to classify these as discordant diagnoses. More precise definition of mantle cell zone B cell and Burkitt lymphomas nowadays may have resulted in the better concordance in our study for these subtypes than in the ILSG study [37 vs. $16 \%(p=0.05)$ and 47 vs. $13 \%(p=0.1)$ ] [4]. In our study, we had no technical problems with cyclin D1 and CD5 immunostaining, which resulted in a statistically significant lower discordance rate in mantle cell lymphoma $(0 \%)$ than in the study performed in India $(30 \%)(p=0.03)[4,6]$.

In our study, all lymphoma cases diagnosed in 2000 and 2001 were reviewed by the panel, although some were excluded from the study because they had been sent for consultation before panel review. However, ideally, in order to reduce the workload of the expert panel and target pathology review to patients who most likely will benefit from it, only cases difficult to classify should be reviewed. We did not know the reasons of the initial pathologists for referring a case to the panel. We assumed that pathologists did not refer a case less often to the panel when they considered the biopsy to be "difficult" to diagnose. Nevertheless, biopsies from patients actively referred to the panel showed a lower discordance rate, compared to the cases not actively referred, although the difference was not significant. This could be caused by the special interest in haematology of some of the initial pathologists who would be more interested in and more aware of the opinion of the expert or perhaps because of differences between laboratories in the policy of referral. To our knowledge, the distinction between referred and not referred lymphoma cases was not made in earlier studies. Even though we cannot reconstruct the reason for not referring a case to the panel, this finding shows that the percentage discordant cases in the panel are not increased by referral bias.

Accurate pathological diagnosis is the essential first step in formulating appropriate treatment strategies. Our study indicates that a panel review was discordant in almost one out of every seven patients, but we did not study whether this actually resulted in different therapy. The study performed in Wales showed that in $46 \%$ of the patients with a discordant diagnosis, therapy was changed [5].

In our study, survival of patients with a discordant diagnosis was not statistically significant different from survival of patients with a concordant diagnosis. We expected that patients with a discordant case probably would have had a worse survival due to suboptimal treatment. However, it is important to realize that the discordant and concordant cases had diverse clinical variables and diagnoses, e.g. different proportion of lymphoma subtypes and a difference in the age distribution, which both affects the survival of lymphoma patients. In addition, seven out of ten lymphomas with a final diagnosis of transformed (composite) lymphoma had an original diagnosis of DLBCL rather than a low-grade lymphoma. Furthermore, other clinical elements (e.g. IPI) were not included in this study. The result that the lowest survival rate was seen in cases actively referred could imply that the referring pathologist knew which cases needed second opinions before initiating therapy, and thus, sent them early for expert review.

Our study had a few limitations that require consideration. The small number of patients per lymphoma subtype limited detailed (survival) analyses by lymphoma subtypes. We expect that the discrepancy and/or delay on a low-grade diagnosis will not have the same impact as a discrepant diagnosis in a high-grade case, but numbers were too limited to show this. Secondly, we did not collect information on the actual therapy received by the patients nor the real change in therapy after the panel diagnosis. Thirdly, only patients with a malignant lymphoma were included in the study. Therefore, patients suspected to have a lymphoma who appeared to have another cancer or no cancer at all were excluded from this study.

This population-based study showed that almost one of seven lymphoma diagnoses was changed by the expert panel. The discordance rate of cases not actively referred to the panel was not lower as compared to cases actively referred. This indicates that pathologists did not actively refer a subset of cases that were difficult to diagnose which presumably would 
have resulted in a higher discordance rate by referral bias. No effect of a discordant diagnosis on survival was observed, probably due to the fact that discrepant cases were a different group compared to concordant cases. Further research is necessary to study the impact of referral and timeliness on survival of specific lymphoma subtypes. From this study, it can be concluded that a panel of expert pathologists results in improved classification of lymphomas.

Acknowledgments We acknowledge the critical review of Dr. Willem Vreuls, Department of Pathology, Canisius-Wilhelmina Hospital, Nijmegen, The Netherlands, of the lymphoma diagnoses in the panel and a final version of the paper.

Conflict of interest The authors declare that they have no conflict of interest.

\section{References}

1. Swerdlow SH, Campo E, Harris NL (2008) WHO Classification of Tumours of Haematopoietic and Lymfoid Tissues. IARC, Lyon

2. Morton LM, Turner JJ, Linet MS et al (2007) Proposed classification of lymphoid neoplasms for epidemiologic research from the Pathology Working Group of the International Lymphoma Epidemiology Consortium (InterLymph). Blood 110(2):695-708

3. Turner JJ, Morton LM, Linet MS et al (2010) InterLymph hierarchical classification of lymphoid neoplasms for epidemiologic research based on the WHO classification (2008): update and future directions. Blood 116:e90-e98

4. A clinical evaluation of the International Lymphoma Study Group classification of non-Hodgkin's lymphoma (1997) The Non-Hodgkin's Lymphoma Classification Project. Blood 89: 3909-3918

5. Lester JF, Dojcinov SD, Attanoos RL et al (2003) The clinical impact of expert pathological review on lymphoma management: a regional experience. Br J Haematol 123:463-468

6. Naresh KN, Agarwal B, Nathwani BN et al (2004) Use of the World Health Organization (WHO) classification of non-Hodgkin's lymphoma in Mumbai, India: a review of 200 consecutive cases by a panel of five expert hematopathologists. Leuk Lymphoma 45:15691577

7. LaCasce AS, Kho ME, Friedberg JW et al (2008) Comparison of referring and final pathology for patients with non-Hodgkin's lymphoma in the National Comprehensive Cancer Network. J Clin Oncol 26:5107-5112

8. Clarke CA, Glaser SL, Dorfman RF et al (2004) Expert review of non-Hodgkin's lymphomas in a population-based cancer registry: reliability of diagnosis and subtype classifications. Cancer Epidemiol Biomarkers Prev 13:138-143

9. Glaser SL, Dorfman RF, Clarke CA (2001) Expert review of the diagnosis and histologic classification of Hodgkin disease in a population-based cancer registry: interobserver reliability and impact on incidence and survival rates. Cancer 92:218-224

10. Sim J, Wright CC (2005) The kappa statistic in reliability studies: use, interpretation, and sample size requirements. Phys Ther 85:257-268

11. Proctor IE, Mcnamara C, Rodriguez-Justo M et al (2011) Importance of Expert Central Review in the Diagnosis of Lymphoid Malignancies in a Regional Cancer Network. J Clin Oncol 29:1431-1435 